\title{
Zygomaticomaxillary Complex Fracture as an Orbital Wall Fracture
}

\author{
Mohammad Waheed El-Anwar* \\ Professor, Otorhinolaryngology, Faculty of Medicine, Head and Neck Surgery, Zagazig University, Egypt
}

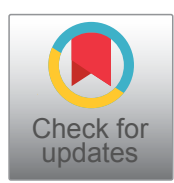

Zygomaticomaxillary complex (ZMC) fracture is common [1-5], involved in approximately $42 \%$ of facial fractures and represents more than $60 \%$ of all middle third fractures [2].

ZMC fracture refers to the osseous disruption of the malar eminence at its 4 buttresses: Frontozygomatic, zygomaticomaxilllary, zygomaticosphenoid and zygomaticotemporal (zygomatic arch) [4]. Thus, ZMC fracture is considered as a type of orbital wall fractures because the lateral orbital wall and orbital floor are important walls that could be affected by such fractures.

The most frequent signs of ZMC fractures are; step deformity of orbital bony margin (100\%), flattening of the cheek (94\%), periorbital ecchymosis (90\%), facial asymmetry (86\%), facial oedema (82\%), epistaxis (68\%) and subconjuntival ecchymosis (52\%) [6].

Particular attention should be given to the eye examination when ZMC fractures are suspected. The anterior and posterior segments of the eye should be assessed, along with evaluation of optic nerve function and ocular motility. Visual field defects decreased visual acuity, abnormal papillary response, or impaired perception of red color saturation is likely indicative of an optic nerve injury. Although uniformly limited mobility may occur secondary to diffuse orbital edema, restricted movement in a vertical orientation is caused by inferior rectus entrapment. Forced diction testing is mandatory in unconscious patients, along with papillary reflex evaluation. Urgent ophthalmological consultation should be obtained in cases have post-trauma diminished visual acuity, abnormal optic nerve findings, sever conjunctival hemorrhage, obvious corneal or globe injury, hyphema, or entrapped foreign body [7].

Computed tomographic (CT) scans ( 1 to $2 \mathrm{~mm}$ slices) from the men tom to the vertex is the standard diagnostic radiology [4] better with 3DCT reconstruction.

ZMC fractures could be treated by observation, closed reduction without fixation or open reduction and rigid internal fixation (OR/IF) at 1 or more ZMC buttresses. One of the most standardized methods for treating ZMC fractures is OR/ IF $[1,3-5]$. Most ZMC fractures (77\% to $94 \%$ ) are surgically repaired $[2,4]$.
Transconjunctival approach for repair of the inferior orbital rim and orbital floor as part of OR/IF of ZMC fractures consume more operative time and mostly needs lateral canthotomy for proper fracture exposure and repair that not needed with subciliary approach. However, transient postoperative edema is more sever in transconjunctival approach but the post-operative ectropion and sclera show is only reported with subciliary approach. So upgrading the surgical experience in transconjuctival approach is beneficial for the maxillofacial surgeons [5].

Although 3 point fixation technique is the conventional standard fixation technique of ZMC fracture [5], 2 points fixation is as effective as 3 points in fixation and prevents post-reduction rotation or clinical displacement with significantly lower cost even with some asymptomatic and clinically unnoticeable radiological difference [8]. Exploration and repair of orbital floor must be conducted during OR/IF o ZMC fractures associated with limited upward gaze.

ZMC fractures significantly reduce muscular activity of the masseter and temporalis muscles that showed significant recovery of muscle activity 6 weeks after repair but this muscle activity remain less than normal activity, reflecting the importance of the postoperative rehabilitation [2] that help to maintain muscle power affecting facial and lid contour.

In spite of disruption of the ZMC bones by its fractures, infraorbital foramen lies just lateral to a line between the anterior nasal spine and whitnal tubercle (clinically nasal tip - lateral canthal ligament line) and lateral to the root of maxillary canine. These fixed reliable landmarks help to avoid mental nerve injury during OR/IF of ZMC fractures [9].

*Corresponding author: Mohammad Waheed El-Anwar, Associate professor, Otorhinolaryngology, Faculty of Medicine, Head and Neck Surgery Department, Zagazig University, Egypt, Tell: 00201004695197, Fax: 0020552307830

Accepted: November 28, 2020

Published online: November 30, 2020

Citation: El-Anwar MW (2020) Zygomaticomaxillary Complex Fracture as an Orbital Wall Fracture. J Head Neck Surg 2(1):9495

Copyright: (C) 2020 El-Anwar MW. This is an open-access article distributed under the terms of the Creative Commons Attribution License, which permits unrestricted use, distribution, and reproduction in any medium, provided the original author and source are credited. 


\section{References}

1. Kurita M, Okazaki M, Ozaki M, et al. (2010) Patient satisfaction after open reduction and internal fixation of zygomatic bone fractures. J Craniofac Surg 21: 45-49.

2. El-Anwar MW, Elsheikh E, Sweed AH, et al. (2015) Electromyography assessment in zygomaticomaxillary complex fractures. Oral Maxillofac Surg 19: 375-379.

3. Yonehara $Y$, Hirabayashi S, Tachi M, et al. (2015) Treatment of zygomatic fractures without inferior orbital rim fixation. J Craniofac Surg 16: 481-485.

4. Meslemani D, Kellman RM (2012) Zygomaticomaxillary complex fractures. arch Facial Plast Surg 14: 62-66.

5. El-Anwar MW, Elsheikh E, Hussein AM, et al. (2017) Transconjunctival versus subciliary approach to the infraorbital margin for open reduction of zygomaticomaxillary complex fractures: A randomized feasibility study. Oral Maxillofac Surg 21: 187-192.

6. Leonardo TA, Moraes RB, Cerqueira JG (2012) Symptomatology of fractures of the zygomatic complex. Analysis of a series of patients. Rev Cir Traumatol Bucco-Maxillo-Fac Camaragibe 12: 73- 80.

7. Soparkar CN, Patrinely JR (2007) The eye examination in facial trauma for the plastic surgeon. Plast Reconstr Surg 120: 49S-56S.

8. Nasr WF, ElSheikh E, El Anwar MW, et al. (2018) Two versus three point internal fixation of displaced zygomaticomaxillary complex fractures. Craniomaxillofac trauma reconstr 11: 256-264.

9. El-Anwar MW, Sweed AH (2018) Inferiororbital foramen localization in orbitozygomatic fractures: A CT study with intraoperative finding. Eur Arch Otorhinolaryngol 275: 809-813. 